\title{
POSITIVE CONES AND FOCAL POINTS FOR A CLASS OF $n$ TH ORDER DIFFERENTIAL EQUATIONS
}

BY

\author{
M. S. KEENER ${ }^{1}$ AND C. C. TRAVIS
}

\begin{abstract}
Necessary and sufficient conditions are obtained for both the existence and absence of focal points for a class of $n$th order linear differential equations. The techniques utilized the theory of $\mu_{0}$-positive operators with respect to a cone in a Banach space.
\end{abstract}

I. Introduction. We shall be concerned with the focality properties on an interval $(\alpha, \beta)$ of a class of $n$th order (possibly nonselfadjoint) differential equations of the form

$$
y^{(n)}(x)-(-1)^{n-k} \sum_{i=0}^{n-1} p_{i}(x) y^{(i)}(x)=0,
$$

where the continuous coefficients $p_{i}$ are assumed to satisfy certain positivity conditions; namely, $p_{i}(x) \geqslant 0,0 \leqslant i \leqslant k,(-1)^{i} p_{k+i}(x) \geqslant 0,1 \leqslant i<n-k$ -1 , for $x \in(\alpha, \beta)$, and $p_{0}$ is not identically zero on any subinterval of $(\alpha, \beta)$. The $(k, n-k)$ focal point $n_{k}(\alpha)$, associated with the point $\alpha$, of the $n$th order differential equation (1.1) is defined to be the smallest real number greater than $\alpha$ such that (1.1) has a solution which is positive on the interval $\left(\alpha, n_{k}(\alpha)\right]$ and satisfies the boundary conditions

$$
\begin{aligned}
y^{(i)}(\alpha) & =0, & & 0 \leqslant i \leqslant k-1, \\
y^{(i)}\left(n_{k}(\alpha)\right) & =0, & & k \leqslant i \leqslant n-1 .
\end{aligned}
$$

If equation (1.1) does not possess a $(k, n-k)$ focal point in the interval $(\alpha, \beta)$, it will be said to be $(k, n-k)$ disfocal on $[\alpha, \beta)$.

The primary purpose of the present study is to investigate the relationship between the existence of $(k, n-k)$ focal points of equation (1.1), and the existence of functions contained in the positive cone of a particular Banach lattice which satisfy certain integral inequalities. Once this relationship has

Received by the editors August 16, 1976.

AMS (MOS) subject classifications (1970). Primary 35C10, 34B10, 34B25, 35B05; Secondary 47B55, 47E05.

Key words and phrases. Linear differential equations, focal points, disfocal, eigenvalues, $\mu_{0}$-positive operators with respect to a cone.

'This research was supported in part by a grant from Oklahoma State University College of Arts and Sciences Office of Research and Graduate Studies. 
been established, it will be employed to obtain explicit criteria, in terms of the coefficients $p_{i}$, for the presence or absence of $(k, n-k)$ focal points of equation (1.1).

While, at first, it may seem more natural to study the equation

$$
y^{(n)}(x)+\sum_{i=0}^{n-1} p_{i}(x) y^{(i)}(x)=0
$$

or the equation

$$
y^{(n)}(x)-\sum_{i=0}^{n-1} p_{i}(x) y^{(i)}(x)=0
$$

we shall demonstrate in §IV that the existence of $(k, n-k)$ focal points for these equations is subject to certain parity restrictions. A necessary condition for an equation of the form (1.3) to have a $(k, n-k)$ focal point is that $n-k$ be odd, while for equations of the form (1.4) $n-k$ must be even. Thus, if we are only concerned with the study of focal points, equations (1.3) and (1.4) may be replaced by the single equation (1.1).

Much of the motivation for the present study comes from results of $\mathbf{Z}$. Nehari [6] on the disconjugacy properties of the equation $y^{(n)}-$ $(-1)^{n-k} p_{0}(x) y=0$. Nehari demonstrated that criteria for the presence or absence of focal points for this class of equations can be formulated in terms of integral inequalities involving appropriate Green's functions. This observation also forms the basis of our results. However, our formulation will be in the abstract setting of a Banach lattice and will rely heavily on the theory of $\mu_{0}$-positive linear operators. Moreover, our method of proof will demonstrate the close relationship between the focality properties of equation (1.1) and the existence of a smallest positive eigenvalue for the $n$th order equation.

$$
y^{(n)}(x)-(-1)^{n-k} \lambda \sum_{i=0}^{n-1} p_{i}(x) y^{(i)}(x)=0
$$

subject to the boundary conditions

$$
\begin{array}{rlrl}
y^{(i)}(\alpha) & =0, & 0 \leqslant i \leqslant k-1, \\
y^{(i)}(\beta) & =0, & & k \leqslant i \leqslant n-k-1 .
\end{array}
$$

This problem will be termed the $(k, n-k)$ focal point eigenvalue problem. A similar, though somewhat less general, eigenvalue problem was studied by $\mathbf{R}$. D. Gentry and C. C. Travis in [1], [2].

In §II we first give some basic results concerning eigenvalues of operators defined on a Banach space $\mathscr{B}$ which are $\mu_{0}$-positive with respect to a cone $\mathscr{P}$. We then introduce the equivalent integral equations for the $(k, n-k)$ focal point eigenvalue problem, and, applying the theory of $\mu_{0}$-positive operators, establish the existence of a smallest positive eigenvalue for the eigenvalue 
problem (1.5)-(1.6). We remark here that without some restriction on the coefficients $p_{i}$, eigenvalue problems of the form (1.5)-(1.6) need not always have an eigenvalue. Indeed, suppose $y(x)$ is a solution of the equation $(\lambda$ finite)

$$
y^{(n)}(x)-(-1)^{n-k} \lambda \sum_{i=k}^{n-1} p_{i}(x) y^{(i)}(x)=0
$$

satisfying conditions (1.6). If we set $v=y^{(k)}$, then $v$ is a solution of

$$
v^{(n-k)}(x)-(-1)^{n-k} \lambda \sum_{i=0}^{n-k-1} p_{k+i}(x) v^{(i)}(x)=0,
$$

with $(n-k)$ zeros at $x=\beta$. Hence $y^{(k)}(x)=v(x) \equiv 0$ on $[\alpha, \beta]$. Using the boundary conditions on $y$ at $x=\alpha$, it follows that $y(x) \equiv 0$ on $[\alpha, \beta]$.

In §III we obtain an extremal characterization of the smallest positive eigenvalue of the $(k, n-k)$ focal point eigenvalue problem. In §IV we obtain necessary and sufficient conditions for the existence or absence of $(k, n-k)$ focal points in terms of integral inequalities involving the Green's function for the operator $(-1)^{n-k^{(n)}}$. In $\S \mathrm{V}$, by considering equation (1.1) under the assumption $p_{i}(x) \equiv 0, k \leqslant i \leqslant n-1$, we obtain more explicit criteria. For example, a sufficient condition for equation $(1.1)$ to be $(n-1,1)$ disfocal on the interval $[0, \infty)$ is that

$$
\begin{aligned}
\frac{1}{(n-1) !} \int_{x}^{\infty} p_{0}(s) s^{n-1} d s+\frac{1}{(n-2) !} \int^{\infty} p_{1}(s) s^{n-2} d s+\ldots & \\
& +\int_{x}^{\infty} p_{n-1}(s) d s \leqslant 1 \text { for } x>0,
\end{aligned}
$$

while a necessary condition that equation $(1.1)$ be $(n-1,1)$ disfocal on the interval $[\alpha, \infty)$ is that

$$
\begin{aligned}
\frac{x}{(n-1) !} \int_{x}^{\infty} p_{0}(s) s^{n-2} d s+\frac{x}{(n-2) !} \int_{x}^{\infty} p(s) s^{n-3} d s+\ldots & \\
& +x \int_{x}^{\infty} p_{n-2}(s) d s<1, \text { for } x \geqslant 0 .
\end{aligned}
$$

II. $\mu_{0}=$ positive operators. Let $\mathscr{B}$ denote a real Banach space.

Definition 2.1. A closed subset $\mathscr{P}$ of $\mathscr{B}$ is called a cone if it satisfies

(i) $u \in \mathscr{P}$ and $v \in \mathscr{P} \Rightarrow u+v \in \mathscr{P}$,

(ii) $u \in \mathscr{P}$ and $\tau \geqslant 0 \Rightarrow \tau u \in \mathscr{P}$,

(iii) $u \in \mathscr{P}$ and $-u \in \mathcal{P} \Rightarrow u=0$.

Every cone $\mathscr{P}$ on a Banach space $\mathscr{T}$ generates a partial ordering on both the space $\mathscr{B}$ and the space of bounded linear operators mapping $\mathscr{B}$ into itself. For a given cone $\mathscr{P}$ of a Banach space $\mathscr{B}$ and elements $u, v \in \mathscr{B}$, we will say that $u<v$ if and only if $v-u \in \mathscr{P}$. For bounded linear operators 
$M$ and $L$ defined on $\mathscr{B}$, we will say that $M<L$ with respect to $\mathscr{P}$ if and only if $M u<L u$ for all $u \in \mathscr{P}$.

Definition 2.2. A bounded linear operator $M$ defined on a Banach space $\mathscr{B}$ is $\mu_{0}$-positive (with respect to a cone $\mathscr{P}$ of $\mathscr{B}$ ) if there exists a nonzero element $\mu_{0} \in \mathcal{P}$ such that for every nonzero element $u \in \mathscr{P}$, there exist positive numbers $k_{1}$ and $k_{2}$ such that $k_{1} \mu_{0}<M u<k_{2} \mu_{0}$.

The class of $\mu_{0}$-positive operators has been studied by M. A. Krasnoselskii and L. A. Ladyzenskii. The interested reader is referred to [4] for an extensive development of their theory and application. The following theorem is one of the fundamental properties of compact $\mu_{0}$-positive linear operators.

THEOREM 2.1. If $L$ is a compact $\mu_{0}$-positive linear operator, then $L$ has exactly one (normalized) eigenvector in $\mathcal{P}$ and the corresponding eigenvalue is simple, positive, and larger than the absolute value of any other eigenvalue.

We next introduce an extension of a result of C. C. Travis [7, p. 365] concerning the comparison of eigenvalues of $\mu_{0}$-positive operators. The proof is similar to that in [7] and will be omitted.

THEOREM 2.2. Let $L$ and $M$ be bounded linear operators and assume that at least one of the operators is $\mu_{0}$-positive. If $L<M$ and

$$
L u_{1}>\lambda_{1} u_{1} \quad\left(u_{1} \in \mathcal{P}, \lambda_{1}>0\right)
$$

and

$$
M u_{2}<\lambda_{2} \mu_{2} \quad\left(u_{2} \in \mathcal{P}, \lambda_{2}>0\right)
$$

then $\lambda_{1}<\lambda_{2}$ and if $\lambda_{1}=\lambda_{2}, u_{1}$ is a scalar multiple of $u_{2}$.

We now introduce the differential operator

$$
D[u]=(-1)^{n-k} u^{(n)}
$$

subject to the $(k, n-k)$ focal point boundary conditions (1.4). It is easily verified that the Green's function for this differential operator is given by the following result.

ThEOREM 2.3. The Green's function $G_{k}$ for the operator (2.1) subject to the $(k, n-k)$ focal point boundary conditions (1.4) is given by

$$
G_{k}(x, s)=\left\{\begin{array}{rr}
\frac{1}{(n-k-1) !} \int_{\alpha}^{x} \int_{\alpha}^{x_{k}} \ldots \int_{\alpha}^{x_{2}}\left(s-x_{1}\right)^{n-k-1} d x_{1} \ldots d x_{k} \\
\frac{1}{(k-1) !} \int_{\alpha}^{x} \int_{\alpha}^{x_{n-k}} \ldots \int_{\alpha}^{x_{2}}\left(x-x_{1}\right)^{k-1} d x_{1} \ldots d x_{n-k} \\
\text { for } \alpha<s<x<\beta, \beta,
\end{array}\right.
$$


or equivalently,

$$
G_{k}(x, s)=\left\{\begin{array}{cc}
\frac{1}{(n-k-1) !} \frac{1}{(k-1) !} \int_{\alpha}^{x}\left(s-x_{1}\right)^{n-k-1}\left(x-x_{1}\right)^{k-1} d x_{1} \\
\frac{1}{(n-k-1) !} \frac{1}{(k-1) !} \int_{\alpha}^{s}\left(s-x_{1}\right)^{n-k-1}\left(x-x_{1}\right)^{k-1} d x_{1} \\
\text { for } \alpha<s<x<\beta .
\end{array}\right.
$$

Rather than studying the differential operator (1.1) subject to the $(k, n-$ $k$ ) focal point boundary conditions (1.4), we introduce the following equivalent integral operator defined on the Banach space

$$
\mathscr{B}=\left\{u \in C^{n-1}[\alpha, \beta] \mid u^{(i)}(\alpha)=0, i=0,1, \ldots, k-1\right\}
$$

equipped with the usual sup norm:

$$
M[u]=\int_{\alpha}^{\beta} G_{k}(x, s) \sum_{i=0}^{n-1} p_{i}(s) u^{(i)}(s) d s .
$$

We shall consider the $\mu_{0}$-positivity of this operator with respect to the cone $\mathscr{P}_{k}$ of functions contained in the Banach space $\mathscr{B}$ such that $u^{(i)}(x)>0$ on $[\alpha, \beta]$ for $i=0,1, \ldots, k$ and $(-1)^{j} u^{(k+j)}(x) \geqslant 0$ on $[\alpha, \beta]$ for $j=0,1, \ldots, n-k$ -1 .

LEMMA 2.4. If $0 \leqslant p_{i}(x)$ on $[\alpha, \beta]$ for $i=0,1, \ldots, k$ and $0 \leqslant(-1)^{j} p_{k+j}(x)$ on $[\alpha, \beta]$ for $j=0,1, \ldots, n-k-1$, then the operator (2.2) is $\mu_{0}$-positive with respect to the cone $\mathscr{P}_{k}$.

Proof. For $v \in \mathscr{P}_{k}$, we shall denote the function $M[v](x)$ by $h(x)$. Now, to demonstrate that the operator $M$ is $\mu_{0}$-positive with respect to the cone $\mathscr{P}_{k}$ we must show that there exists $\mu_{0} \in \mathscr{P}_{k}$ such that for any nonzero $v \in \mathscr{P}_{k}$, there exist positive constants $r_{1}$ and $r_{2}$ such that

$$
\begin{gathered}
r_{1} \mu_{0}^{(i)}(x) \leqslant h^{i}(x) \leqslant r_{2} \mu_{0}^{(i)}(x), \quad i=0,1, \ldots, k, \\
r_{1}(-1)^{j} \mu_{0}^{(k+j)}(x) \leqslant(-1)^{j} h^{(k+j)}(x) \leqslant r_{2}(-1)^{j} \mu_{0}^{(k+j)}(x), \\
j=0,1, \ldots, n-k-1 .
\end{gathered}
$$

Let $v$ be a fixed but arbitrary nonzero element of the cone $\mathscr{P}_{k}$ and let $\mu_{0}$ be the function defined by

$$
\mu_{0}(x)=\int_{\alpha}^{\beta} G_{k}(x, s) d s .
$$

Since we are assuming that $v \in \mathscr{P}_{k}$, we have that $\sum_{i=0}^{n-1} p_{i}(x) v^{(i)}(x)>0$ on $[\alpha, \beta]$. Thus for $i=0,1, \ldots, k$, we have 


$$
\mu_{0}^{(i)}(x)=\int_{\alpha}^{\beta} \frac{\partial^{i}}{\partial x^{i}} G_{k}(x, s) d s \geqslant 0
$$

and

$$
h^{(i)}(x)=\int_{\alpha}^{\beta} \frac{\partial^{i}}{\partial x^{i}} G_{k}(s, s) \sum_{i=0}^{n-1} p_{i}(s) v^{(i)}(s) d s \geqslant 0 .
$$

Also, since

$$
\mu_{0}^{(k)}(x)=\frac{1}{(n-k-1) !} \int_{x}^{\beta}(s-x)^{n-k-1} d s
$$

and

$$
h^{(k)}(x)=\frac{1}{(n-k-1) !} \int_{x}^{\beta}(s-x)^{n-k-1} \sum_{i=0}^{n-1} p_{i}(s) v^{(i)}(s) d s,
$$

we have that for $j=0,1, \ldots, n-k-1$,

$$
(-1)^{j} \mu_{0}^{(k+j)}(x)=\frac{1}{(n-k-j-1) !} \int_{x}^{\beta}(s-x)^{n-k-j-1} d s>0
$$

and

$$
\begin{aligned}
& (-1)^{j} h^{(k+j)}(x) \\
& \quad=\frac{1}{(n-k-j-1) !} \int_{x}^{\beta}(s-x)^{n-k-j-1} \sum_{i=0}^{n-1} p_{i}(s) v^{(i)}(s) d s>0 .
\end{aligned}
$$

Thus $\mu_{0} \in \mathscr{P}_{k}$ and the operator $M$ is positive with respect to the cone $\mathscr{P}_{k}$. We will now show that $M$ is $\mu_{0}$-positive where $\mu_{0}$ is defined by (2.4). It follows from the above calculation that

$$
(-1)^{n-k} \mu_{0}^{(n)}(x) \equiv 1
$$

and

$$
(-1)^{n-k} h^{(n)}(x)=\sum_{i=0}^{n-1} p_{i}(x) v^{(i)}(x),
$$

and hence that $(-1)^{n-k} \mu_{0}^{(n)}(\beta)>0$ and $(-1)^{n-k} h^{(n)}(\beta)>0$. As $\mu_{0}^{(n)}$ and $h^{(n)}$ are continuous, we may determine positive constants $\varepsilon_{1}$ and $\varepsilon_{2}$ and $a \xi \in$ $[\alpha, \beta)$ such that, for $x \in[\xi, \beta]$,

$$
\varepsilon_{1}(-1)^{n-k} \mu_{0}^{(n)}(x)<(-1)^{n-k} h^{(n)}(x)<\varepsilon_{2}(-1)^{n-k} \mu_{0}^{(n)}(x) .
$$

Integrating this inequality and using the fact that $\mu_{0}^{(n-1)}(\beta)=h^{(n-1)}(\beta)=0$ yields the inequalities,

$$
\varepsilon_{1}(-1)^{n-k-1} \mu_{0}^{(n-1)}(x)<(-1)^{n-k-1} h^{(n-1)}(x)<\varepsilon_{2}(-1)^{n-k-1} \mu_{0}^{(n-1)}(x)
$$

for $x \in[\xi, \beta]$. Now, from the fact that $(-1)^{n-k-1} \mu_{0}^{(n)}(x)<0$ and 
$(-1)^{n-k-1} h^{(n)}(x) \leqslant 0$ on $[\alpha, \beta]$ and the fact that $\mu_{0}^{(n-1)}(\xi)$ and $h^{(n-1)}(\xi)$ are positive, we conclude that $(-1)^{n-k-1} \mu_{0}^{(n-1)}(x)$ and $(-1)^{n-k-1} h^{(n-1)}(x)$ are strictly positive and bounded on $[\alpha, \xi]$. Consequently, we may determine positive constants $\delta_{1}$ and $\delta_{2}$ such that for $x \in[\alpha, \xi]$,

$$
\delta_{1}(-1)^{n-k-1} \mu_{0}^{(n-1)}(x) \leqslant(-1)^{n-k-1} h^{(n-1)}(x) \leqslant \delta_{2}(-1)^{n-k-1} \mu_{0}^{(n-1)}(x) .
$$

Setting $r_{1}=\operatorname{minimum}\left\{\varepsilon_{1}, \delta_{1}\right\}$ and $r_{2}=\operatorname{maximum}\left\{\varepsilon_{2}, \delta_{2}\right\}$, we have that

$$
r_{1}(-1)^{n-k-1} \mu_{0}^{(n-1)}(x) \leqslant(-1)^{n-k-1} h^{(n-1)}(x) \leqslant r_{2}(-1)^{n-k-1} \mu_{0}^{(n-1)}(x)
$$

for all $x$ in $[\alpha, \beta]$. Integrating these inequalities and using the fact that $\mu_{0}$ and $h$ satisfy the boundary conditions

$$
\begin{aligned}
\mu_{0}(\alpha) & =\mu_{0}^{\prime}(\alpha)=\cdots=\mu_{0}^{(k-1)}(\alpha)=\mu_{0}^{(k)}(\beta) \\
& =\mu_{0}^{(k+1)}(\beta)=\cdots=\mu_{0}^{(n-1)}(\beta)=0
\end{aligned}
$$

and

$$
\begin{aligned}
h(\alpha) & =h^{\prime}(\alpha)=\cdots=h^{(k-1)}(\alpha)=h^{(k)}(\beta) \\
& =h^{(k+1)}(\beta)=\cdots=h^{(n-1)}(\beta)=0,
\end{aligned}
$$

we obtain (2.3) and the proof is complete.

An application of Ascoli's theorem enables one to demonstrate that the operator $M$ is compact on the Banach space $\mathscr{B}$. Thus it follows from Theorem 2.1 that $M$ has exactly one normalized eigenvector in the cone $\mathscr{P}_{k}$ and the corresponding eigenvalue is simple, positive, and larger than the absolute value of any other eigenvalue. In order to express this result in terms of differential equations, consider the equation

$$
\begin{aligned}
& u^{(n)}(x)-(-1)^{n-k} \lambda \sum_{i=0}^{n-1} p_{i}(x) u^{(i)}(x)=0, \\
& 0<p_{i}(x) \text { on }[\alpha, \beta] \text { for } i=0,1, \ldots, k, \\
& 0<(-1)^{j} p_{k+j}(x) \text { on }[\alpha, \beta] \text { for } j=0,1, \ldots, n-k-1,
\end{aligned}
$$

subject to the boundary conditions

$$
\begin{aligned}
u(\alpha) & =u^{\prime}(\alpha)=\cdots=u^{(k-1)}(\alpha)=0, \\
u^{(k)}(\beta) & =u^{(k+1)}(\beta)=\cdots=u^{(n-1)}(\beta)=0 .
\end{aligned}
$$

A similar, though somewhat less general, eigenvalue problem was studied by R. D. Gentry and C. C. Travis in [1]. Our previous discussion establishes the following theorem concerning the existence of a smallest positive eigenvalue for this problem.

THEOREM 2.4. The $(k, n-k)$ focal point eigenvalue problem (2.5)-(2.6) has at least one real eigenvalue which is simple, positive and smaller than the 
absolute value of any other eigenvalue. The normalized eigenvector associated with this eigenvalue is contained in the cone $\mathscr{P}_{k}$ and is the only eigenvector with this property.

III. An extremal principle. In this section we are interested in obtaining an extremal characterization of the smallest positive eigenvalue of $\lambda_{0}$ of the $n$th order nonselfadjoint $(k, n-k)$ focal point eigenvalue problem

$$
\begin{aligned}
& u^{(n)}(x)-(-1)^{n-k} \lambda \sum_{i=0}^{n-1} p_{i}(x) u^{(i)}(x)=0, \\
& u(\alpha)=u^{\prime}(\alpha)=\cdots=u^{(k-1)}(\alpha)=0, \\
& u^{(k)}(\beta)=u^{(k+1)}(\beta)=\cdots=u^{(n-1)}(\beta)=0,
\end{aligned}
$$

where the coefficients $p_{i}$ satisfy the positivity conditions $0<p_{0}(x)$ on $[\alpha, \beta]$, $0 \leqslant p_{i}(x)$ on $[\alpha, \beta]$ for $i=1,2, \ldots, k$, and $0 \leqslant(-1)^{j} p_{k+j}(x)$ on $[\alpha, \beta]$ for $j=1,2, \ldots, n-k-1$.

The Banach space $\mathscr{B}$, the cone $\mathscr{P}_{k}$, and the bounded linear operator $M$ are assumed to be defined as in $\$ I I$. We shall let $\mathscr{B}^{\prime}$ denote the dual space of continuous linear functionals on $\mathscr{B}$, and $\mathscr{P}_{k}^{\prime}$ denote the "dual cone" of all elements of $\mathscr{B}^{\prime}$ that are nonnegative on $\mathscr{P}_{k}$, i.e. $\mathscr{P}_{k}^{\prime}=\left\{x^{\prime} \in \mathscr{B}^{\prime}:\left\langle x, x^{\prime}\right\rangle>0\right.$ for all $\left.x \in \mathscr{P}_{k}\right\}$, where $\left\langle x, x^{\prime}\right\rangle$ denotes the pairing between an element $x^{\prime}$ of $\mathscr{B}^{\prime}$ and $x$ of $\mathscr{B}$. Since $\mathscr{P}_{k}$ is closed, it follows from the Krein-Putnam theorem, on the positive extension of a positive linear functional from a subspace to the whole space, that from the relation $\left.\left\langle x, x^{\prime}\right\rangle\right\rangle 0$ for all $x^{\prime} \in \mathscr{P}_{k}^{\prime}$, one can conclude that $x \in \mathscr{P}_{k}$. We shall also have need of the spectral radius of $M$, i.e., $\sup \{|\lambda| \mid \lambda \in \sigma(M)\}$, which we shall denote by $r(M)$. We note that since $M$ is compact $\lambda_{0}^{-1}=r(M)$.

LEMMA 3.1. If $0 \leqslant p_{0}(x)$ on $[\alpha, \beta], 0 \leqslant p_{i}(x)$ on $[\alpha, \beta]$ for $i=1,2, \ldots, k$, $0<(-1)^{j} p_{k+j}(x)$ on $[\alpha, \beta]$ for $j=1,2, \ldots, n-k-1$, and $r(M)<\lambda$, then $(\lambda I-M)^{-1}$ is a bounded operator which is positive with respect to the cone $\mathscr{P}_{k}$.

Proof. That $(\lambda I-M)^{-1}$ is a bounded operator follows from the definition of the spectral radius of $M$. To demonstrate that $(\lambda I-M)^{-1}$ is a positive operator with respect to the cone $\mathscr{P}_{k}$, we first notice that it follows from Lemma 2.1 that $M$ is positive with respect to the cone $\mathscr{P}_{k}$. Now, since the cone $\mathscr{P}_{k}$ is closed, we have that for any $x \in \mathscr{P}_{k}$,

$$
(\lambda I-M)^{-1} x=\sum_{n=1}^{\infty} \lambda^{-n} M^{N-1} x \in \mathscr{P}_{k} .
$$

We are now ready to present the main result of this section.

THEOREM 3.1. If $0<p_{0}(x)$ on $[\alpha, \beta], 0 \leqslant p_{i}(x)$ on $[\alpha, \beta]$ for $i=$ $1,2, \ldots, k$, and $0 \leqslant(-1)^{j} p_{k+j}(x)$ on $[\alpha, \beta]$ for $j=1,2, \ldots, n-k-1$, 
then the smallest positive eigenvalue $\lambda_{0}$ of the $(k, n-k)$ focal point eigenvalue problem (3.1) can be characterized by the extremal principle

$$
\lambda_{0}^{-1}=\max _{\substack{u \in P_{k} \\ u \neq 0}} \frac{\int_{\alpha}^{\beta} \int_{\alpha}^{\beta} G_{k}(x, s) u(x) \sum_{i=0}^{n+1} p_{i}(s) u^{(i)}(s) d s d x}{\int_{\alpha}^{\beta} u^{2}(x) d x} .
$$

The unique function, except for a constant multiple, which maximizes (3.2) is the positive eigenfunction corresponding to the eigenvalue $\lambda_{0}$.

Proof. Define the mapping $\xi: \mathscr{P}_{\boldsymbol{k}} \rightarrow \mathbf{R}$ by

$$
\xi(x)=\left\{\begin{array}{l}
\sup \left\{\lambda \in \mathbf{R} \mid \lambda\left\langle x, x^{\prime}\right\rangle \leqslant\left\langle M x, x^{\prime}\right\rangle, x^{\prime} \in \mathcal{P}_{k}^{\prime}\right\} \quad \text { if } x \neq 0, \\
0 . \text { if } x=0 .
\end{array}\right.
$$

We first notice that $0 \leqslant \xi(x)$, since $M$ is positive with respect to $\mathscr{P}_{k}$. However, in order to show that $\xi$ is well defined, we must demonstrate that for $x \in \mathscr{P}_{k}, \sup \left\{\lambda \in \mathbf{R} \mid \lambda\left\langle x, x^{\prime}\right\rangle \leqslant\left\langle M x, x^{\prime}\right\rangle, x^{\prime} \in \mathscr{P}_{k}^{\prime}\right\}<\infty$. We shall do this by demonstrating that $\xi(x) \leqslant r(M)$ for all $x \in \mathscr{P}_{k}$. Suppose that there exists $x_{0} \in \mathscr{P}_{k}$ such that $r(M)<\xi\left(x_{0}\right) \leqslant+\infty$. Then there exists $\mu>r(M)$ such that $\mu\left\langle x_{0}, x^{\prime}\right\rangle\left\langle\left\langle M x_{0}, x^{\prime}\right\rangle\right.$ for all $x^{\prime} \in \mathscr{P}_{k}^{\prime}$. This implies that $M x_{0}-\mu x_{0}$ $\in \mathscr{P}_{k}$, from which it follows that

$$
-x_{0}=(\mu I-M)^{-1}(M-\mu I) x_{0} \in \mathscr{P}_{k} \text {. }
$$

Thus $x_{0}=0$, which is a contradiction. We have now shown that $0<\xi(x)<$ $r(M)$ for all $x \in \mathscr{P}_{k}$ and hence that

$$
\sup _{x \in \Phi_{k}} \xi(x) \leqslant r(M) .
$$

Now, for each $u \in \mathscr{P}_{k}$, define the positive linear functional $u^{\prime} \in \mathscr{P}_{k}^{\prime}$ by $\left\langle x, u^{\prime}\right\rangle=\int_{\alpha}^{\beta} x(s) u(s) d s$ for all $x \in \mathscr{P}_{k}$. Then, for each $x \in \mathscr{P}_{k}$, we have that $\sup \left\{\lambda \mid \lambda\left\langle x, u^{\prime}\right\rangle\left\langle\left\langle M x,{ }^{\prime} u\right\rangle\right\}<\xi(x)\right.$ and, consequently, that $\sup \{\lambda \mid$ $\lambda\left\langle u, u^{\prime}\right\rangle\left\langle\left\langle M u,{ }^{\prime} u\right\rangle\right\} \leqslant \xi(u)<r(M)$. It follows that

$$
\sup _{\substack{u \in \Phi_{k} \\ u \neq 0}} \frac{\int_{\alpha}^{\beta}[M u](s) u(s) d s}{\int_{\alpha}^{\beta} u^{2}(s) d s} \leqslant r(M)=\lambda_{0}^{-1} .
$$

Since we have equality when $u$ is equal to a constant multiple of the eigenfunction corresponding to $\lambda_{0}$, the first part of the theorem is proven.

To prove the remainder of the theorem, we shall let $\mu_{0}$ denote the positive normalized eigenfunction corresponding to the eigenvalue $\lambda_{0}$ and assume that there exists $\hat{u} \neq 0 \in \mathscr{P}_{k}$ such that

$$
\int_{\alpha}^{\beta}[M \hat{u}](s) \hat{u}(s) d s / \int_{\alpha}^{\beta} \hat{u}^{2}(s) d s=\lambda_{0}^{-1} .
$$

We would like to show that $\hat{u}$ is a scalar multiple of $\mu_{0}$. Let $\lambda_{n} \in \mathbf{R}$ be a sequence such that $\lambda_{n} \rightarrow \xi(\hat{u})$ and $0<M \hat{u}-\lambda_{n} \hat{u}$. Since the cone is closed, 
$M \hat{u}-\xi(\hat{u}) \hat{u}=\lim _{n \rightarrow \infty} M \hat{u}-\lambda_{n} \hat{u} \in \mathscr{P}_{k}$. It follows from the definition of $\xi$ that $\xi(\hat{u})=\lambda_{0}^{-1}$ and thus that $\lambda_{0}^{-1} \hat{u} \leqslant M \hat{u}$. We already know that $M u_{0}=$ $\lambda_{0}^{-1} u_{0}$ and that $M$ is $\mu_{0}$-positive with respect to the cone $\mathscr{P}_{k}$. We can thus apply Theorem 2.2 to conclude that $\hat{u}$ is a scalar multiple of $u_{0}$.

COROLLARY 3.1. The smallest positive eigenvalue $\lambda_{0}$ of the eigenvalue problem (3.1) is a strictly monotone decreasing function of the right end point $\beta$ of the integral $[\alpha, \beta]$.

Proof. Let $\lambda_{0}\left(\beta_{i}\right), i=1,2$, denote the smallest positive eigenvalue of (3.1) on the interval $\left[\alpha, \beta_{i}\right], i=1,2$. We must demonstrate that $\beta_{1}<\beta_{2}$ implies that $\lambda_{0}\left(\beta_{2}\right)<\lambda_{0}\left(\beta_{1}\right)$. Let $v_{1}$ denote the the normalized eigenfunction corresponding to the eigenvalue $\lambda_{0}\left(\beta_{1}\right)$. Define the function $h \in C^{n-1}\left[\alpha, \beta_{2}\right]$ by

$$
h(x)= \begin{cases}v_{1}(x) & \text { for } x \in\left[\alpha, \beta_{1}\right], \\ \sum_{i=0}^{k-1} \frac{\left(x-\beta_{1}\right)^{i}}{i !} v_{1}^{(i)}\left(\beta_{1}\right) & \text { for } x \in\left[\beta_{1}, \beta_{2}\right] .\end{cases}
$$

Then $h$ is contained in the positive cone $\mathscr{P}_{k}$ for $\mathscr{B}=C^{n-1}\left[\alpha, \beta_{2}\right]$. We know that

$$
\lambda_{0}^{-1}\left(\beta_{1}\right) v_{1}^{(k)}(x)=\frac{1}{(n-k-1) !} \int_{x}^{\beta_{1}}(s-x)^{n-k-1} \sum_{i=0}^{n-1} p_{i}(s) v_{1}^{(i)}(s) d s
$$

for $x \in\left[\alpha, \beta_{1}\right]$, and, since

$$
h^{(k)}(x)= \begin{cases}v_{1}^{(k)}(x) & \text { for } x \in\left[\alpha, \beta_{1}\right], \\ 0 & \text { for } x \in\left[\beta_{1}, \beta_{2}\right],\end{cases}
$$

we have that

$$
\lambda_{0}^{-1}\left(\beta_{1}\right) h^{(k)}(x)<\frac{1}{(n-k-1) !} \int_{x}^{\beta_{2}}(s-x)^{n-k-1} \sum_{i=0}^{n-1} p_{i}(s) h^{(i)}(s) d s
$$

for $x \in\left[\alpha, \beta_{2}\right]$. Now if we let

$$
R(x)=\int_{\alpha}^{\beta_{2}} G_{k}(x, s) \sum_{i=0}^{n-1} p_{i}(s) h^{(i)}(s) d s
$$

for $x \in\left[\alpha, \beta_{2}\right]$, then

$$
\begin{aligned}
\lambda_{0}^{-1}\left(\beta_{1}\right) h^{(k)}(x) & <\frac{1}{(n-k-1) !} \int_{x}^{\beta_{2}}(s-x)^{n-k-1} \sum_{i=0}^{n-1} p_{i}(s) h^{(i)}(s) d s \\
& =R^{(k)}(x)
\end{aligned}
$$

for $x \in\left[\alpha, \beta_{2}\right]$. Since $R-\lambda_{0}^{-1}\left(\beta_{1}\right) h$ is of class $C^{k-1}[\alpha, \beta]$, by Taylor's Theorem 


$$
\begin{aligned}
R(x)-\lambda_{0}^{-1}\left(\beta_{1}\right) h(x) & =\sum_{i=0}^{k-1} \frac{\left[R^{(i)}(\alpha)-\lambda_{0}^{-1}\left(\beta_{1}\right) h^{(i)}(\alpha)\right]}{i !}(x-\alpha)^{i} \\
+ & \frac{1}{(k-1) !} \int_{\alpha}^{x}(x-s)^{k-1}\left[R^{(k)}(s)-\lambda_{0}^{-1}\left(\beta_{1}\right) h^{(k)}(s)\right] d s .
\end{aligned}
$$

Since $R^{(i)}(\alpha)=h^{(i)}(\alpha)=0$ for $i=0,1, \ldots,-1$, it follows that $\lambda_{0}^{-1}\left(\beta_{1}\right) h(x)$ $<R(x)$ for $x \in\left[\alpha, \beta_{2}\right]$. Thus

$$
\begin{aligned}
\lambda_{0}^{-1}\left(\beta_{1}\right) & <\int_{\alpha}^{\beta_{2}} G_{k}(x, s) h(x) \sum_{i=0}^{n-1} p_{i}(j) h^{(i)}(s) d s / \int_{\alpha}^{\beta_{2}} h^{2}(s) d s \\
& \leqslant \max _{\substack{\mu \in \Phi_{k} \\
\mu \neq 0}} \int_{\alpha}^{\beta_{2}} G_{k}(x, s) \mu(x) \sum_{i=0}^{n-1} p_{i}(s) \mu^{(i)}(s) d s / \int_{\alpha}^{\beta_{2}} \mu^{2}(s) d s \\
& =\lambda_{0}^{-1}\left(\beta_{2}\right) .
\end{aligned}
$$

The result now follows.

IV. Focal points. We first review the definition of the $(k, n-k)$ focal point $n_{k}(\alpha)$ associated with the point $\alpha$. It is defined to be the smallest real number greater than $\alpha$ such that the $n$th order differential equation

$$
u^{(n)}(x)-(-1)^{n-k} \sum_{i=0}^{n-1} p_{i}(x) u^{(i)}(x)=0
$$

has a solution which is positive on the interval $\left(\alpha, n_{k}(\alpha)\right]$ and satisfies the conditions

$$
\begin{gathered}
u(\alpha)=u^{\prime}(\alpha)=\cdots=u^{(k-1)}(\alpha)=0, \\
u^{(k)}(\beta)=u^{(k+1)}(\beta)=\cdots=u^{(n-1)}(\beta)=0,
\end{gathered}
$$

where $\beta=n_{k}(\alpha)$. The equation (4.1) is termed $(k, n-k)$ disfocal on the interval $[\alpha, \beta)$ if it does not possess a $(k, n-k)$ focal point in this interval. Our first result establishes a relationship between the absence of a $(k, n-k)$ focal point of equation (4.1) and the magnitude of the smallest positive eigenvalue $\lambda_{0}$ of the $(k, n-k)$ focal point eigenvalue problem

$$
u^{(n)}(x)=(-1)^{n-k} \lambda \sum_{i=0}^{n-1} p_{i}(x) u^{(i)}(x)=0,
$$

subject to the boundary conditions (4.2).

THEOREM 4.1. A necessary and sufficient condition for equation (4.1) to be $(k, n-k)$ disfocal on the interval $[\alpha, \beta), \alpha<\beta \leqslant+\infty$, is that the smallest eigenvalue $\lambda_{0}(\bar{\beta})$ of the eigenvalue problem (4.3) on the interval $[\alpha, \beta]$ satisfy $1<\lambda_{0}(\bar{\beta})$, for every $\bar{\beta}$ such that $\alpha<\bar{\beta}<\beta$.

Proof. If equation (4.1) has a $(k, n-k)$ focal point $\beta_{1}=n(\alpha)$ in the 
interval $[\alpha, \beta)$, then the smallest positive eigenvalue of (4.3) on the interval $\left[\alpha, \beta_{1}\right]$ is $\lambda_{0}\left(\beta_{1}\right)=1$. Since $\lambda_{0}(\beta)$ is monotonic decreasing as a function of the endpoint $\beta$ of the interval $[\alpha, \beta]$, it follows that $\lambda_{0}(\bar{\beta})<1$ for $\beta_{1}<\bar{\beta}$. To prove the converse, assume that equation (4.1) is $(k, n-k)$ disfocal on the interval $[\alpha, \beta)$ and that there exists a $\bar{\beta}$ such that $\alpha<\bar{\beta}<\beta$ and $\lambda_{0}(\bar{\beta})<1$. As $\beta$ decreases to $\alpha$, the eigenvalue $\lambda_{0}(\beta)$ increases without limit in a continuous manner. There exists therefore a value $\beta_{1}\left(\alpha<\beta_{1}<\bar{\beta}\right)$ for which $\lambda_{0}\left(\beta_{1}\right)=1$. This is in contradiction to the fact that equation (4.1) is $(k, n-$ $k)$ disfocal on the interval $[\alpha, \beta)$. Since the smallest positive eigenvalue $\lambda_{0}(\beta)$ is monotonic decreasing as a function of $\beta$, it follows that a necessary and sufficient condition for equation (4.1) to be $(k, n-k)$ disfocal on a finite interval $[\alpha, \beta)$ is that $1<\lambda_{0}(\beta)$. This completes the proof of the theorem.

We know from the definition of the $(k, n-k)$ focal point $n_{k}(\alpha)$ that the corresponding $(k, n-k)$ focal function is positive on $\left(\alpha, n_{k}(\alpha)\right]$. Actually, much more is true: the $(k, n-k)$ focal function belongs to the cone $\mathscr{P}_{k}$. To see this, let $\beta=n_{k}(\alpha)$ be the $(k, n-k)$ focal point associated with the point $\alpha$, and $v$ the corresponding $(k, n-k)$ focal function. Then $v$ satisfies the equation

$$
v(x)=\int_{\alpha}^{\beta} G_{k}(x, s) \sum_{i=0}^{n-1} p_{i}(s) v^{(i)}(s) d s .
$$

We know that the $(k, n-k)$ focal point eigenvalue problem on the interval $[\alpha, \beta]$ has a smallest positive eigenvalue $\lambda_{0}$ and an eigenfunction $u_{0}(x)$ belonging to the cone $\mathcal{P}_{k}$ which satisfies

$$
\lambda_{0}^{-1} u_{0}(x)=\int_{\alpha}^{\beta} G_{k}(x, s) \sum_{i=0}^{n-1} p_{i}(s) u_{0}^{(i)}(s) d s .
$$

If $\lambda_{0}<1$, then it follows from Theorem 4.1 that equation (4.1) has a $(k, n-k)$ focal point in the interval $[\alpha, \beta)$, which is impossible. Thus $1<\lambda_{0}$. If $1<\lambda_{0}$ then $\lambda_{0}^{-1}$ would not be the largest eigenvalue of the compact $\mu_{0}$-positive operator

$$
M[u]=\int_{\alpha}^{\beta} G_{k}(x, s) \sum_{i=0}^{n-1} p_{i}(s) u^{(i)}(s) d s,
$$

in contradiction to Theorem 2.1. Thus $\lambda_{0}=1$ and $v$ is a constant multiple of $u_{0}$, and hence is contained in the cone $\mathscr{P}_{k}$.

Since every $(k, n-k)$ focal function actually belongs to the cone $\mathscr{P}_{k}$, we shall at this point modify our definition of a $(k, n-k)$ focal point. The $(k, n-k)$ focal point $n_{k}(\alpha)$ shall be defined as the smallest real number greater than $\alpha$ such that the $n$th order differential equation (4.1) has a nontrivial solution contained in the cone $\mathscr{P}_{k}$ which satisfies the boundary conditions (4.2). 
Nehari [6] has shown that the $(k, n-k)$ focal points of equations of the form

$$
u^{(n)}(x)+P(x) u(x)=0, \quad P(x)>0,
$$

or

$$
u^{(n)}(x)-P(x) u(x)=0, \quad P(x)>0,
$$

are subject to certain parity restrictions: for equation (4.5), $n-k$ must be odd, while for equation (4.6) $n-k$ must be even. A similar result is true for equations of the form (4.1). In fact, if equation

$$
u^{(n)}(x)+\sum_{i=0}^{n-1} p_{i}(x) u^{(i)}(x)=0
$$

were to have a $(k, n-k)$ focal point with $n-k$ even, then the $(k, n-k)$ focal function $v$ would satisfy

$$
-v(x)=\int_{\alpha}^{\beta} G_{k}(x, s) \sum_{i=0}^{n-1} p_{i}(s) v^{(i)}(s) d s .
$$

This is impossible, since the right side of this equation is positive while the left side is negative. We thus have the following result.

THEOREM 4.2. A necessary condition for the equation

$$
u^{(n)}(x)+\sum_{i=0}^{n-1} p_{i}(x) u^{(i)}(x)=0,
$$

to have $a(k, n-k)$ focal point is that $n-k$ be odd, while a necessary condition for the equation

$$
u^{(n)}(x)-\sum_{i=0}^{n-1} p_{i}(x) u^{(i)}(x)=0,
$$

to have $a(k, n-k)$ focal point is that $n-k$ be even.

Thus, if we are only concerned with the study of $(k, n-k)$ focal points, equations (4.7) and (4.8) may be replaced by the single equation (4.1). At this point, we shall present some necessary and sufficient conditions for the absence of $(k, n-k)$ focal points on a finite interval $[\alpha, \beta)$ which are formulated in terms of integral inequalities involving the Green's function $G_{k}(x, s)$. These results extend corresponding results by Nehari [6].

THEOREM 4.3. A necessary and sufficient condition for equation (4.1) to be $(k, n-k)$ disfocal on the finite interval $[\alpha, \beta)$ is that there exists a nontrivial function $v$ contained in the cone $\mathscr{P}_{k}$ such that

$$
\int_{\alpha}^{\beta} G_{k}(\cdot, s) \sum_{i=0}^{n-1} p_{i}(s) v^{(i)}(s) d s \leqslant v
$$


with respect to the cone $\mathscr{P}_{k}$. That is, for $i=0,1, \ldots, k$,

$$
\int_{\alpha}^{\beta} \frac{\partial i}{\partial x^{i}} G_{k}(x, s) \sum_{i=0}^{n-1} p_{i}(s) v^{(i)}(s) d s<v^{(i)}(x)
$$

and, for $j=0,1, \ldots, n-k-1$,

$$
\begin{gathered}
\frac{1}{(n-k-j-1) !} \int_{x}^{\beta}(s-x)^{n-k-j-1} \sum_{i=0}^{n-1} p_{i}(s) v^{(i)}(s) d s \\
<(-1)^{j} v^{(k+j)}(x)
\end{gathered}
$$

for $x$ in $[\alpha, \beta]$.

Proof. If equation (4.1) is $(k, n-k)$ disfocal on the interval $[\alpha, \beta)$, then by Theorem 4.1 , the smallest positive eigenvalue $\lambda_{0}(\beta)$ of the eigenvalue problem (4.3) satisfies $1<\lambda_{0}(\beta)$. Let $v$ be the normalized eigenfunction contained in the cone $\mathscr{P}_{k}$ corresponding to $\lambda_{0}(\beta)$. Then

$$
\int_{\alpha}^{\beta} G_{k}(\cdot, s) \sum_{i=0}^{n-1} p_{i}(s) v^{(i)}(s) d s=\lambda_{0}^{-1}(\beta) v<v
$$

with respect to the cone $\mathscr{P}_{k}$. To prove the converse, suppose that there exists a nontrivial function $v$ contained in the cone $\mathscr{P}_{k}$ such that

$$
M[v]=\int_{\alpha}^{\beta} G_{k}(\cdot, s) \sum_{i=0}^{n-1} p_{i}(s) v^{(i)}(s) d s<v
$$

with respect to the cone $\mathscr{P}_{k}$. Letting $\lambda_{0}(\beta)$ be the smallest positive eigenvalue of equation (4.3) and $w$ its corresponding normalized eigenfunction, we have $M[w]=\lambda_{0}^{-1}(\beta) w$. Since $M$ is a compact $\mu_{0}$-positive operator, it follows from Theorem 2.2 that $1<\lambda_{0}(\beta)$, and hence that equation (4.1) is $(k, n-k)$ disfocal on the interval $[\alpha, \beta)$.

COROLlary 4.4. A necessary and sufficient condition for equation (4.1) to be $(k, n-k)$ disfocal on the finite interval $[\alpha, \beta)$ is that there exists a nontrivial function $v$ contained in the cone $\mathscr{P}_{k}$ such that

$$
(-1)^{n-k} v^{(n)}(x)-\sum_{i=0}^{n-1} p_{i}(x) v^{(i)}(x) \geqslant 0
$$

for $x$ in $[\alpha, \beta]$.

Proof. If equation (4.1) is $(k, n-k)$ disfocal on the interval $[\alpha, \beta)$, then the normalized eigenfunction $v$ corresponding to the smallest positive eigenvalue $\lambda_{0}(\beta)$ of the eigenvalue problem (4.3) satisfies

$$
v^{(n)}(x)-(-1)^{n-k} \lambda_{0}(\beta) \sum_{i=0}^{n-1} p_{i}(x) v^{(i)}(x)=0,
$$


for $x$ in $[\alpha, \beta]$. Thus

$$
\sum_{i=0}^{n-1} p_{i}(x) v^{(i)}(x)=\lambda_{0}^{-1}(\beta)(-1)^{n-k} v^{(n)}(x)<(-1)^{n-k} v^{(n)}(x),
$$

for $x$ in $[\alpha, \beta]$, since $\lambda_{0}^{-1}(\beta)<1$. To prove the converse, assume that there exists a nontrivial function $v$ contained in the cone $\mathscr{P}_{k}$ such that (4.13) is satisfied. Repeated integration of (4.13) from $x$ to $\beta$, combined with the fact that $(-1)^{j} v^{(k+j)}(\beta) \geqslant 0$ for $j=0,1, \ldots, n-k-1$, yields (4.11). Repeated integration, over the interval $[\alpha, x]$, of

$$
\frac{1}{(n-k-1) !} \int_{x}^{\beta}(s-x)^{n-k-1} \sum_{i=0}^{n-1} p_{i}(s) v^{(i)}(s) d s<v^{(k)}(x),
$$

combined with the fact that $v^{(i)}(\alpha)=0$ for $i=0,1, \ldots, k-1$, yields (4.9). Thus by Theorem 4.3, equation (4.1) is $(k, n-k)$ disfocal on the interval $[\alpha, \beta)$, and the proof is complete.

This corollary may be used to provide a comparison result.

COROLlary 4.5. If equation (4.1) is $(k, n-k)$ disfocal on the finite interval $[\alpha, \beta)$ and if the continuous functions $\left\{q_{i}\right\}_{i=0}^{n-1}$ satisfy the inequalities

$$
\begin{gathered}
q_{i}(x) \leqslant p_{i}(x), \quad i=0,1, \ldots, k, \\
(-1)^{i} q_{k+i}(x) \leqslant(-1)^{i} p_{k+i}(x), \quad i=1, \ldots, n-k-1,
\end{gathered}
$$

on $[\alpha, \beta]$, then the equation

$$
u^{(n)}-(-1)^{n-k} \sum_{i=0}^{n-1} q_{i}(x) u^{(i)}=0
$$

is $(k, n-k)$ disfocal on $[\alpha, \beta)$.

Proof. If equation (4.1) is $(k, n-k)$ disfocal on the interval $[\alpha, \beta)$, then by Corollary 4.4 there exists a nontrivial function $v \in \mathscr{P}_{k}$ such that

$$
(-1)^{n-k} v^{(n)}(x)>\sum_{i=0}^{n-1} p_{i}(x) v^{(i)}(x) \text { on }[\alpha, \beta] .
$$

Since $\sum_{i=0}^{n-1} p_{i}(x) v^{(i)}(x)>\sum_{i=0}^{n-1} q_{i}(x) v^{(i)}(x)$ on $[\alpha, \beta]$, the proof is completed by applying the sufficiency criteria of Corollary 4.4.

We are also able to obtain necessary and sufficient conditions for the existence of $(k, n-k)$ focal points on a finite interval $(\alpha, \beta]$ in terms of integral inequalities involving the Green's function $G_{k}$.

THEOREM 4.6. $A$ necessary and sufficient condition for the interval $(\alpha, \beta]$ to contain a $(k, n-k)$ focal point of equation (4.1) is that there exists a nontrivial function $v$ contained in the cone $\mathscr{P}_{k}$ such that 


$$
\int_{\alpha}^{\beta} G_{k}(\cdot, s) \sum_{i=0}^{n-1} p_{i}(s) v^{(i)}(s) d s \geqslant v
$$

with respect to the cone $\mathscr{P}_{k}$. That is, for $i=0,1, \ldots, k$,

$$
\int_{\alpha}^{\beta} \frac{\partial^{i}}{\partial x^{i}} G_{k}(x, s) \sum_{i=0}^{n-1} p_{i}(s) v^{(i)}(s) d s>v^{(i)}(x),
$$

and for $j=0,1, \ldots, n-k-1$,

$$
\begin{gathered}
\frac{1}{(n-k-j-1) !} \int_{x}^{\beta}(s-x)^{n-k-j-1} \sum_{i=0}^{n-1} p_{i}(s) v^{(i)}(s) d s \\
\geqslant(-1)^{j} v^{(k+j)}(x),
\end{gathered}
$$

for $x$ in $[\alpha, \beta]$.

Proof. If equation (4.1) has a $(k, n-k)$ focal point $n_{k}(\alpha)=\beta_{1}$ in the interval $(\alpha, \beta]$, then the smallest positive eigenvalue $\lambda_{0}\left(\beta_{1}\right)$ of the eigenvalue problem (4.3) on the interval $\left[\alpha, \beta_{1}\right]$ satisfies $\lambda_{0}\left(\beta_{1}\right)=1$. Since $\beta_{1}<\beta$, we have that $\lambda_{0}(\beta)<\lambda_{0}\left(\beta_{1}\right)=1$. Let $v$ be the normalized eigenfunction contained in the cone $\mathscr{P}_{k}$ corresponding to $\lambda_{0}(\beta)$. Then

$$
\int_{\alpha}^{\beta} G_{k}(\cdot, s) \sum_{i=0}^{n-1} p_{i}(s) v^{(i)}(s) d s=\lambda_{0}^{-1}(\beta) v \geqslant v
$$

with respect to the cone $\mathscr{P}_{k}$. Now, suppose that the inequality (4.14) is satisfied for some nontrivial $v$ contained in the cone $\mathscr{P}_{k}$. Let $\lambda_{0}(\beta)$ be the smallest positive eigenvalue of the eigenvalue problem (4.3) and $w$ the corresponding normalized eigenfunction. Then $M[w]=\lambda_{0}^{-1}(\beta) w$ and $M[v]$ $>v$. Since $M$ is a compact $\mu_{0}$-positive operator, Theorem 2.2 implies that $\lambda_{0}(\beta)<1$, and it thus follows from Theorem 4.1 that equation (4.1) has a $(k, n-k)$ focal point $n_{k}(\alpha)$ in the interval $(\alpha, \beta]$.

We present the following corollary without proof:

COROLlary 4.7. A necessary and sufficient condition for equation (4.1) to have $a(k, n-k)$ focal point in the interval $(\alpha, \beta]$ is that there exists a nontrivial function $v$ contained in the cone $\mathscr{P}_{k}$ such that

$$
(-1)^{n-k} v^{(n)}(x)-\sum_{i=0}^{n-1} p_{i}(x) v^{(i)}(x) \leqslant 0
$$

for $x$ in $[\alpha, \beta]$.

V. More on focal points. In this section we consider the $(k, n-k)$ focal point problem for the less general equation

$$
y^{(n)}-(-1)^{n-k} \sum_{i=0}^{k} p_{i}(x) u^{(i)}(x)=0
$$


where $p_{i}, i=0,2, \ldots, k$, are nonnegative and continuous on $[\alpha, \beta]$. Since the higher order derivatives are missing in equation (5.1) it is possible to obtain more explicit conditions for disfocality than those presented in §IV. Furthermore, these results generalize those of Nehari [6].

The problem may be formulated as above by considering the differential operator $D u=(-1)^{n-k} u^{(n)}$, subject to the $(k, n-k)$ focal point boundary conditions (1.2). We use the Green's function (Theorem 2.3) to define the integral operator

$$
M[u]=\int_{\alpha}^{\beta} G_{k}(x, s) \sum_{i=0}^{k} p_{i}(s) u^{(i)}(s) d s
$$

on the Banach space $C^{k}[\alpha, \beta]$. Following the same analysis as in $\S I$ II, $M$ is a compact $\mu_{0}$-positive operator with respect to the cone $2_{k}$ of function in $C^{k}[\alpha, \beta]$ for which $u^{(i)}(x) \geqslant 0$ on $[\alpha, \beta], i=0,1, \ldots, k$. Note that the only essential difference in the two formulations is the choice of the cones. It follows that the results of $\S I V$ also hold for equation (5.1). For reference we state the analogues of Theorems 4.3 and 4.6 respectively.

THEOREM 5.1. A necessary and sufficient condition for equation (5.1) to be $(k, n-k)$ disfocal on the finite interval $[\alpha, \beta)$ is that there exists a nontrivial function $v$ contained in the cone $2_{k}$ such that

$$
\int_{\alpha}^{\beta} \frac{\partial^{i}}{\partial x^{i}} G_{k}(x, s) \sum_{i=0}^{k} p_{i}(s) v^{(i)}(s) d s<v^{(i)}(x)
$$

for $i=0,1,2, \ldots, k$ and $x \in[\alpha, \beta]$.

THEOREM 5.2. A necessary and sufficient condition for the interval $(\alpha, \beta]$ to contain $a(k, n-k)$ focal point for equation (5.1) is that there exists a nontrivial function $v$ contained in the cone $\mathscr{2}_{k}$ such that for $x \in[\alpha, \beta]$,

$$
\int_{\alpha}^{\beta} \frac{\partial^{i}}{\partial x^{i}} G_{k}(x, s) \sum_{i=0}^{k} p_{i}(s) v^{(i)}(s) d s>v^{(i)}(x)
$$

for $i=0,1, \ldots, k$.

The following simpler characterizations can now be given which generalize Theorem 5.2 [6].

THEOREM 5.3. Equation (5.1) is $(k, n-k)$ disfocal on the finite interval $[\alpha, \beta]$ if and only if there exists a nontrivial function $v \in \mathscr{2}_{k}$ such that for $x \in[\alpha, \beta]$,

$$
\frac{1}{(n-k-1) !} \int_{x}^{\beta}(s-x)^{n-k-1} \sum_{i=0}^{k} p_{i}(s) v^{(i)}(s) d s \leqslant v^{(k)}(x) .
$$

Proof. Assuming that equation (5.1) is $(k, n-k)$ disfocal on $[\alpha, \beta)$, the 
result follows trivially from Theorem 5.1. Suppose that such a function $v \in \mathscr{2}_{k}$ exists. Integrating the quality (5.4) from $\alpha$ to $x$ and using the $(k, n-k)$ focal point boundary conditions (1.2), it follows from Theorem 5.1 that equation (5.1) is $(k, n-k)$ disfocal on $[\alpha, \beta)$, and the proof is complete.

Due to Theorem 5.3, we may obtain an explicit sufficient condition on the coefficients $p_{i}, i=0,1, \ldots, k$, for equation (5.1) to be $(k, n-k)$ disfocal. For example, define $v(x)=(x-\alpha)^{k} / k !$. Then $v^{(i)}(x)=(x-\alpha)^{k-i} /(k-$ $i) !, i=0,1, \ldots, k$, and $v^{(i)}(x) \equiv 0, i>k$. Then $v \in \mathscr{Q}_{k}$ and condition (5.4) becomes

$$
\begin{aligned}
& \frac{1}{(n-k-1) !} \int_{x}^{\beta}(s-x)^{n-k-1} \sum_{i=0}^{k} \frac{p_{i}(s)}{(k-i) !}(s-\alpha)^{k-i} d s \leqslant 1, \\
& x \in[\alpha, \beta] .
\end{aligned}
$$

This proves the following corollary.

COROLlary 5.4. Condition (5.5) is sufficient for equation (5.1) to be ( $k, n-$ $k)$ disfocal on $[\alpha, \beta)$.

It easily follows that a sufficient condition for equation (5.1) to be ( $k, n-$ $k$ ) disfocal on $(\alpha, \infty)$ is that for $x \in[\alpha, \infty)$

$$
\frac{1}{(n-k-1) !} \int_{x}^{\infty}(s-x)^{n-k-1} \sum_{i=0}^{k} \frac{p_{i}(s)}{(k-i) !}(s-\alpha)^{k-i} d s<1 .
$$

If we further restrict our attention to the Banach lattice $\mathscr{B}=\{u \in$ $C^{(k-1)}[\alpha, \beta] \mid u^{(i)}(\alpha)=0$ for $\left.i=0,1, \ldots, k-1\right\}$ with cone $2_{k-1}=\{u \in$ $\mathscr{B} \mid u^{(i)}>0$ for $\left.i=0,1, \ldots, k-1\right\}$, we can find an explicit necessary condition in terms of the coefficients for the $(k, n-k)$ disfocality on the interval $[\alpha, \beta)$ of the equation

$$
u^{(n)}(x)-(-1)^{n-k} \sum_{i=0}^{k-1} p_{i}(x) u^{(i)}(x)=0
$$

We first reformulate the sufficient condition of Theorem 5.2 as it applies to equation (5.6).

THEOREM 5.5. A sufficient condition for the interval $[\alpha, \beta)$ to contain a $(k, n-k)$ focal point of equation (5.6) is that there exists a nontrivial function $v$ contained in the cone $2_{k-1}$ such that

$$
\int_{\alpha}^{\beta} G_{k}(\cdot, s) \sum_{i=0}^{k-1} p_{i}(s) v^{(i)}(s) d s \geqslant v
$$

with respect to the cone $\mathscr{2}_{k-1}$.

The following corollary follows trivially from Theorem 5.5. 
COROllary 5.6. A sufficient condition for the interval $(\alpha, \beta]$ to contain a $(k, n-k)$ focal point of equation (5.6) is that there exists a nontrivial function $v$ with the following properties:

(i) $v \in C^{(k-1)}[\alpha, \beta]$ and $v \in \mathscr{Q}_{k-1}$,

(ii) $v^{(k)}$ exists and is continuous on $[\alpha, \beta]$ except for a finite number of discontinuities,

(iii) $(n-k-1) !^{-1} \int_{x}^{\beta}(s-x)^{n-k-1} \sum_{i=0}^{k-1} p_{i}(s) v^{(i)}(s) d s \geqslant v^{(k)}(x)$ for $\alpha<x$ $<\beta$.

As an application of Corollary 5.6 we present the following necessary condition for the absence of focal points. The proof is based on a technique of Nehari [6].

THEOREM 5.7. A necessary condition for equation (5.6) to be $(k, n-k)$ disfocal on the finite interval $[\alpha, \beta)$ is that for $x \in[\alpha, \beta)$,

$$
\begin{aligned}
\frac{1}{(n-k-1) !} \int_{x}^{\beta}(s-x)^{n-k-1} \sum_{i=0}^{k-1} \frac{p_{i}(s)}{(k-i-1) !} \\
\quad \cdot \int_{\alpha}^{x}(s-t)^{k-i-1} d t d s \leqslant 1 .
\end{aligned}
$$

PROOF. For fixed but arbitrary $x \in[\alpha, \beta)$, define

$$
w(s)= \begin{cases}\frac{1}{(k-1) !} \int_{\alpha}^{s}(s-t)^{k-1} d t, & \alpha \leqslant s \leqslant x<\beta, \\ \frac{1}{(k-1) !} \int_{\alpha}^{x}(s-t)^{k-1} d t, & \alpha \leqslant x<s \leqslant \beta .\end{cases}
$$

For each $x \in[\alpha, \beta), w \in \mathscr{Q}_{k-1}$ and

$$
w^{(k)}(s)= \begin{cases}1, & \alpha \leqslant s \leqslant x<\beta \\ 0, & \alpha \leqslant x<s \leqslant \beta\end{cases}
$$

For $t \in[\alpha, \beta]$, define

$$
h(t)=w^{(k)}(t)-\frac{1}{(n-k-1) !} \int_{t}^{\beta}(s-t)^{n-k-2} \sum_{i=0}^{k-1} p_{i}(s) w^{(i)}(s) d s .
$$

If $t \neq x$, then this differentiable and

$$
h^{\prime}(t)=\frac{1}{(n-k-2) !} \int_{t}^{\beta}(s-t)^{n-k-1} \sum_{i=0}^{k-1} p_{i}(s) w^{(i)}(s) d s>0,
$$

and furthermore $h(x-)-h(x+)=1$. Since $h(\beta)=0, h(t) \leqslant 0$ for all $t \in[x, \beta]$, and, since $h(t)>0$ on $[\alpha, x), h(x)<0$ would imply $h(t)<0$ for $t \in[\alpha, x)$. It follows that if $h(x) \leqslant 0$, then $h(t) \leqslant 0$ for all $t \in[\alpha, \beta]$, i.e., for $t \in[\alpha, \beta]$, 


$$
w^{(k)}(t) \leqslant \frac{1}{(n-k-1) !} \int_{t}^{\beta}(s-t)^{n-k-1} \sum_{i=0}^{k-1} p_{i}(s) w^{(i)}(s) d s .
$$

Applying Corollary 5.6, $(\alpha, \beta]$ would contain a $(k, n-k)$ focal point for equation (5.6). Since the equation is assumed to be $(k, n-k)$ disfocal on $[\alpha, \beta)$, we must have $h(x) \geqslant 0$, or equivalently, condition (5.7) holds. This completes the proof of the theorem.

To obtain a necessary condition for equation (5.6) to be $(k, n-k)$ disfocal on the interval $[\alpha, \infty)$ we note that (5.7) must hold for all $\beta>\alpha$. In particular we obtain

$$
\begin{aligned}
\frac{1}{(n-k-1) !} \int_{x}^{\infty}(s-x)^{n-k-1} \sum_{i=0}^{k-1} \frac{p_{i}(s)}{(k-i-1) !} \\
\quad \int_{\alpha}^{x}(s-t)^{k-i-1} d t d s<1 .
\end{aligned}
$$

If we apply condition (5.8) to the equation

$$
y^{(n)}+\sum_{i=0}^{n-2} p_{i}(x) y^{(i)}=0
$$

where $0<p_{i}, i=0,1, \ldots, n-2$, then, since $s^{n-i-1}-(s-x)^{n-i-1}>$ $s^{n-i-2} x$, a necessary condition for equation $(5.9)$ to be $(n-1,1)$ disfocal on the interval $[0, \infty)$ is that

$$
\sum_{i=0}^{n-2} \int_{x}^{\infty} \frac{p_{i}(s)}{(n-i-1) !} s^{n-i-2} x d s<1 ;
$$

or

$$
x \sum_{i=0}^{n-2} \frac{(n-1) !}{(n-i-1) !} \int_{x}^{\infty} s^{n-i-2} p_{i}(s) d s<(n-1) ! .
$$

For $n=2$, the condition (5.11) reduces to the well-known sharp necessity condition of Hille [3] for the equation $y^{\prime \prime}+p_{0}(x) y=0$ to be disfocal on $[0, \infty)$, namely

$$
x \int_{x}^{\infty} p_{0}(s) d s<1
$$

Furthermore, if we consider the equation $(5.6)$ with $p_{i}(x) \equiv 0$ on $[0, \infty)$ for $i=1,2, \ldots, n-2$, then we have the equation $y^{(n)}+p_{0}(x) y=0$ considered by Nehari [6]. Condition (5.11) becomes

$$
x \int_{x}^{\infty} s^{n-2} p_{0}(s) d s \leqslant(n-1) !, \quad x \in(0, \infty),
$$

which is precisely the condition obtained by Nehari for this equation. Finally we note that when condition (5.11) is applied to the equation 


$$
y^{(n)}+p(x) y^{(n-j)}=0, \quad 0 \leqslant j<n-2,
$$

it yields

$$
x \int_{x}^{\infty} s^{j-2} p(s) d s<(j-1) !
$$

as a necessary condition for $(n-1,1)$ disfocality.

\section{BIBLIOGRAPHY}

1. R. D. Gentry and C. C. Travis, Comparison of eigenvalues associated with linear differential equations of arbitrary order, Trans. Amer. Math. Soc. 223 (1976) 167-178.

2. Existence and comparison of eigenvalues of nth order linear differential equations, Bull. Amer. Math. Soc. 82 (1976), 350-352.

3. E. Hille, Non-oscillation theorems, Trans. Amer. Math. Soc. 64 (1948), 234-252. MR 10, 376.

4. M. A. Krasnosel'skii, Positive solutions of operator equations, Fizmatgiz, Moscow, 1962; English transl., Noordhoff, Groningen, 1964. MR 26 \#2862; 31 \#6107.

5. Z. Nehari, Disconjugate linear differential operators, Trans. Amer. Math. Soc. 129 (1967), 500-516. MR 36 \#2860.

6. __ Green's functions and disconjugacy, (to appear).

7. C. C. Travis, Comparison of eigenvalues for linear differential equations of order $2 n$, Trans. Amer. Math. Soc. 177 (1973), 363-374. MR 47 \#5356.

Department of Mathematics, OkLahoma State Universtty, Stmlwater, OxLahoma 74074 (Current address of M. S. Keener)

Department of Mathematics, University of Tennessee, Knoxville, Tennessee 37916

Current address (C. C. Travis): Oak Ridge National Laboratory, Oak Ridge, Tennessee 37839 